PREPRINT

\title{
Macroion adsorption: The crucial role of excluded volume and coions
}

\author{
René Messina* \\ Institut für Theoretische Physik II, \\ Heinrich-Heine-Universität Düsseldorf, \\ Universitätsstrasse 1, D-40225 Düsseldorf, Germany
}

(Dated: October 28, 2018)

\begin{abstract}
The adsorption of charged colloids (macroions) onto an oppositely charged planar substrate is investigated theoretically. Taking properly into account the finite size of the macroions, unusual behaviors are reported. It is found that the role of the coions (the little salt-ions carrying the same sign of charge as that of the substrate) is crucial to understand the mechanisms involved in the process of macroion adsorption. In particular, the coions can accumulate near the substrate's surface and lead to a counter-intuitive surface charge amplification.

PACS numbers: 68.43.De, 68.08.-p, 82.70.Dd, 61.20.Gy
\end{abstract}

Typeset by REVTEX 


\section{INTRODUCTION}

Whereas the bulk behavior of homogeneous ( $\operatorname{charged}^{1,2}$ and uncharged ${ }^{3}$ ) colloidal suspensions is rather well understood, the situation for its inhomogeneous counterpart, such as that emerging in an adsorption process, is less clear. Potential applications of adsorption of charged colloidal particles (macroions) can vary from technological processes such as surface coating $\stackrel{4}{4}$ to biological material problems. $\stackrel{5}{-5}$ From a fundamental point of view, the tremendous long-ranged Coulomb interaction that sets in represents a formidable theoretical challenge. Consequently, a deeper understanding of the phenomenon of macroion adsorption is justified and needed.

On one hand, experiments $\frac{6.7}{7}$ and the well known mean field Gouy-Chapman theory ${ }^{8}, \underline{9}$ seem to nicely agree for the ion distribution of an aqueous monovalent electrolyte near planar charged interfaces, as long as non-specific forces as well as excluded volume effects are negligible. On the other hand, if the solution contains highly multivalent and/or large sized ions, then the Gouy-Chapman theory may severely qualitatively fail. A crucial missing ingredient in this theory is the inclusion of the finite size of the macroions that can lead to non-trivial phenomena, such as substrate's surface charge reversal, already with monovalent ions $\underline{10}, 11,12,13$ The lateral macroion-macroion electrostatic correlations that are also absent in a mean field theory can be attenuated (and even become marginal) at sufficiently high salt content, in contrast to excluded volume effects. In the past, Gonzáles-Mozuelos and Medina-Noyola ${ }^{14}$ used integral equations to address the problem of macroions near repulsive/attractive charged walls interacting via an effective Yukawa potential. More recently, Netz ${ }^{15}$ investigated thoroughly and analytically the behavior of macroions near charged interfaces, based on the Debye-Hückel (DH) theory, but ignoring its finite size. Thereby, the striking effect of surface charge amplification advocated here could not be captured

by those approaches. $\stackrel{14,15}{ }$ It is only very recently, that this phenomenon was reported by

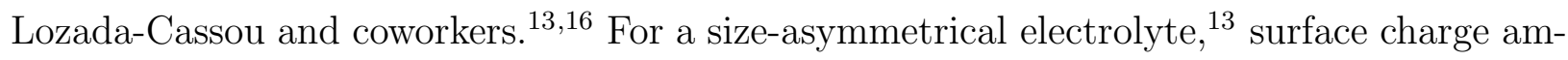
plification was identified by solving numerically the modified Gouy-Chapman (where cations and anions have different distances of closest approach to the interface). Intimately related to our work, the phenomenon of surface charge amplification in presence of macroions was also found by applying a sophisticated hypernetted chain/mean spherical approximation (HNC/MSA) integral equation. ${ }^{16}$ 
In this work, we present a simple model, where the finite size of the macroions is taken into account, to reveal the mechanisms governing macroion adsorption. Although our approach is less accurate than the one used by Lozada-Cassou and coworkers, $\frac{16}{1}$ it presents the nice advantage to be analytical and very intuitive. The basic driving force of surface charge amplification is that (spherical) macroions tend to be surrounded by its counterions over its whole surface in a uniform manner. This corresponds actually to the old classical Thomson's sphere problem. 17 As long as the strength of the surface charge density of the oppositely charged substrate is low enough, a finite number of counterions of the macroions should stay in the vicinity of the interface (see Fig. 1), leading to a surface charge amplification. Our paper is organized as follows: Our (modified) DH theory is explained in Sec. II] The results are presented in Sec. III and followed by a brief summary (see Sec. IV]).

\section{DEBYE HÜCKEL THEORY}

\section{A. Linearized Poisson-Boltzmann equation}

Our electrostatic model (see Fig. 1) resembles that employed by Spitzer $\underline{10}$ who studied monovalent size-asymmetrical ions near a wall. The negative substrate's surface charge density is denoted by $\sigma_{0}$. The macroions carry a positive central charge $+Z_{m} e$ with $e$

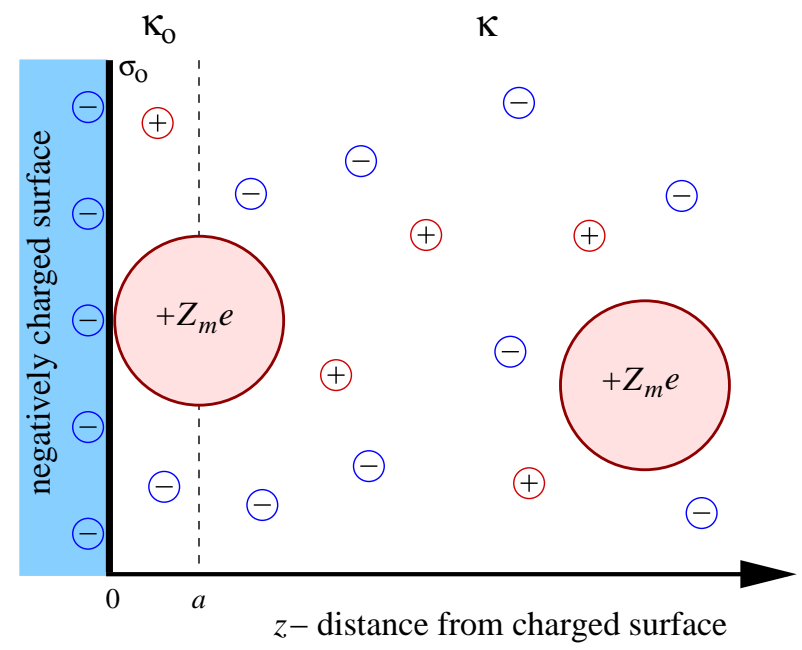

FIG. 1: Schematic view of our model setup. The macroions are characterized by a distance of closest approach $z=a$ to the charged surface, leading to two screening strengths $\kappa_{0}$ and $\kappa$ for $0<z<a$ and $z>a$, respectively. 
representing the usual elementary charge and $Z_{m}$ its valency. Excluded volume effects are taken into account via the distance of closest approach $a$ to the charged interface (see Fig. 1). Our model corresponds somehow to the minimal correction for macroion' size effect. Having to deal with a finite concentration of macroions at contact with a reservoir, whose bulk value is given by $n_{m}$, electroneutrality requires a bulk macroion's counterion concentration $Z_{m} n_{m}$ (assuming monovalent point-like anions, i.e. $Z_{-}=-1$ ). Those anions will be referred to as the coions of the substrate. Additional (point-like) monovalent counterions $\left(Z_{+}=+1\right)$ and coions are also considered with a bulk salt concentration $n_{s}$.

An intuitive and widely used way to connect self-consistently the electrostatic potential, $\Psi(z)$, to the total charge density, $\rho(z) \approx \sum_{\alpha} n_{\alpha} Z_{\alpha} e \exp \left(-\beta Z_{\alpha} e \Psi\right)$ (with $\alpha$ standing for the ionic species), is provided by the so-called Poisson-Boltzmann equation that reads:

$$
\begin{aligned}
\Delta \Psi(z)= & -\frac{e}{\varepsilon_{0} \varepsilon_{r}}\left[-2 n_{s} \sinh (e \beta \Psi)-Z_{m} n_{m} \exp (e \beta \Psi)\right. \\
& \left.+Z_{m} n_{m} \exp \left(-Z_{m} e \beta \Psi\right) \Theta(z-a)\right],
\end{aligned}
$$

where $\varepsilon_{0}\left(\varepsilon_{r}\right)$ is the vacuum (relative) permittivity, $\beta=1 /\left(k_{B} T\right)$ is the reduced inverse temperature, and $\Theta$ is the usual step (or Heaviside) function. This non-linear differential equation (1) can only be solved numerically. However a linearization of Eq. (1) [i.e., DH approximation], valid for $|e \beta \Psi| \ll 1$ when $0<z<a$ and for $\left|Z_{m} e \beta \Psi\right| \ll 1$ when $z>a$, permits an analytical treatment that is going to be discussed.

For the first diffuse region $(0<z<a)$ made up uniquely of the little monovalent ions (see also Fig. 1), the DH equation is given by $\Delta u(z)=\left(\kappa_{s}^{2}+\kappa_{c}^{2}\right) u+\kappa_{c}^{2}$ with $\kappa_{s}^{2} \equiv 8 \pi \ell_{B} n_{s}$ and $\kappa_{c}^{2} \equiv 4 \pi \ell_{B} Z_{m} n_{m}$, where we have introduced the Bjerrum length $\ell_{B}=\frac{e^{2}}{4 \pi \varepsilon_{0} \varepsilon_{r} k_{B} T}$ and the dimensionless variable $u=e \beta \Psi$. The corresponding solution reads:

$$
u(z)=C_{1} e^{-\kappa_{0} z}+C_{2} e^{\kappa_{0} z}-\frac{\kappa_{c}^{2}}{\kappa_{0}^{2}} \quad(0<z<a),
$$

where $\kappa_{0}^{2} \equiv \kappa_{s}^{2}+\kappa_{c}^{2}=4 \pi \ell_{B}\left(2 n_{s}+Z_{m} n_{m}\right) . C_{1}$ and $C_{2}$ are integration constants that are going to be determined after having applied the suitable boundary conditions.

For the second diffuse region $(z>a)$ containing all the ions (including the macroions see also Fig. (1) the DH equation reads $\Delta u(z)=\kappa^{2} u$ with $\kappa^{2} \equiv \kappa_{0}^{2}+Z_{m} \kappa_{c}^{2}=4 \pi \ell_{B}\left[2 n_{s}+\right.$ $\left.Z_{m}\left(Z_{m}+1\right) n_{m}\right]$. The physically sound solution with vanishing electric field at $z \rightarrow+\infty$ corresponds to:

$$
u(z)=C_{3} e^{-\kappa z} \quad(z>a),
$$


where $C_{3}$ is a third integration constant.

\section{B. Boundary conditions}

Our electrostatic model system is completely characterized once the integration constants $C_{1}, C_{2}$ and $C_{3}$ appearing in Eqs. (21) and (3) are specified. To do so, we apply the three following boundary and/or matching conditions: (i) The Gauss' law applied at the charged interface $z=0$ requires $u^{\prime}(0)=\frac{2}{b}$, where $b=\frac{e}{2 \pi \ell_{B}\left|\sigma_{0}\right|}$ is the so-called Gouy-Chapman length. (ii) The zero intrinsic surface charge at $z=a$ imposes the continuity of the electric displacement $\vec{D}=\varepsilon_{0} \varepsilon_{r} \vec{E}$ and hence also that of the electric field $\vec{E}$ [i.e., $u^{\prime}\left(z \rightarrow a^{-}\right)=$ $\left.u^{\prime}\left(z \rightarrow a^{+}\right)\right] \stackrel{18}{\underline{18}}$ (iii) The continuity of the electrostatic potential at $z=a$ requires $u(z \rightarrow$ $\left.a^{-}\right)=u\left(z \rightarrow a^{+}\right)$. The resulting set of three equations can be readily solved and yields:

$$
\begin{gathered}
C_{1}=\frac{-2 Q-\frac{2}{\kappa_{0} b} e^{\kappa_{0} a}+\frac{\kappa_{c}^{2}}{\kappa_{0}^{2}}}{2 \cosh \left(\kappa_{0} a\right)}, \\
C_{2}=\frac{-2 Q-\frac{2}{\kappa_{0} b} e^{\kappa_{0} a}+\frac{\kappa_{c}^{2}}{\kappa_{0}^{2}}}{2 \cosh \left(\kappa_{0} a\right)}+\frac{2}{\kappa_{0} b}, \\
C_{3}=-2 e^{\kappa a} \underbrace{\left[\frac{\frac{1}{\kappa_{0} b \cosh \left(\kappa_{0} a\right)}+\frac{\kappa_{c}^{2}}{2 \kappa_{0}^{2}} \tanh \left(\kappa_{0} a\right)}{\frac{\kappa}{\kappa_{0}}+\tanh \left(\kappa_{0} a\right)}\right]}_{\equiv Q} .
\end{gathered}
$$

Note that in the limit of point-like macroions $\left(\kappa_{0} a \rightarrow 0\right)$ one recovers the well-known result $C_{3} \rightarrow-\frac{2}{\kappa b}$. At this stage all the relevant observables of the system can be in principle obtained within the framework of the DH theory.

\section{RESULTS AND DISCUSSION}

A pertinent quantity that is appropriate to characterize the strength of the macroion adsorption is provided by the contact potential of interaction $U_{m}$. The latter corresponds to the external work accomplished upon bringing a macroion from infinity $(z=+\infty)$ to contact $(z=a): U_{m}=-\int_{+\infty}^{a} Z_{m} e E_{z} d z$ (with $E_{z}=-\frac{\partial \Psi}{\partial z}$ denoting the $z$-component of the electric field). With the help of Eq. (3) we get $\beta U_{m}=Z_{m} u(a)=Z_{m} C_{3} e^{-\kappa a}$. Using then Eq. 
(6) for $C_{3}$, we obtain the following expression for $U_{m}$ :

$$
\beta U_{m}=-2 Z_{m}\left[\frac{\frac{1}{\kappa_{0} b \cosh \left(\kappa_{0} a\right)}+\frac{\kappa_{c}^{2}}{2 \kappa_{0}^{2}} \tanh \left(\kappa_{0} a\right)}{\frac{\kappa}{\kappa_{0}}+\tanh \left(\kappa_{0} a\right)}\right] .
$$

As can be seen from Eq. (7), $U_{m}$ depends on many parameters, such as $Z_{m}, b, a, \kappa$ and $\kappa_{0}, \frac{19}{9}$ making its understanding rather difficult. Nonetheless, Eqs. (21) - (17) suggest that more insight on the adsorption behavior can be gained by considering two limits: (i) the high screening regime $\left(\kappa_{0} a \gg 1\right)$ and (ii) the weak screening regime $\left(\kappa_{0} a \ll 1\right)$. Without loss of generality, we are going to explore these two limits.

\section{A. High screening regime}

In the high screening regime $\left(\kappa_{0} a \gg 1\right)$, Eq. (7) can be approximated by:

$$
\beta U_{m} \simeq 1-\frac{\kappa}{\kappa_{0}}
$$

where the relation $\kappa_{c}^{2}=\frac{1}{Z_{m}}\left(\kappa^{2}-\kappa_{0}^{2}\right)$ has been used. Interestingly, in this regime, $U_{m}$ depends only on the screening strength contrast $\kappa / \kappa_{0}$. One can also conveniently express $U_{m}$ as a function of the bulk concentrations $n_{m}$ and $n_{s}$, which reads

$$
\beta U_{m} \simeq 1-\sqrt{\frac{2 n_{s}+Z_{m}\left(Z_{m}+1\right) n_{m}}{2 n_{s}+Z_{m} n_{m}}} .
$$

Equation (9) shows that $U_{m}$ is even independent of the Bjerrum length $\ell_{B}$, which means that the effective wall-macroion attraction is entropically (or depletion) driven. To better understand this phenomenon, we have sketched on Fig. 2 the profile of $u(z)$ as well as profile of the reduced (dimensionless) electric field $E^{*}(z) \equiv-\frac{b}{2} u^{\prime}(z)$ [with $E^{*}(z=0)=-1$ ] for a set of typical parameters of charged colloidal suspensions: $a=0.5 \mu \mathrm{m}, b=10^{-3} \mu \mathrm{m}$ (corresponding to $\left|\sigma_{0}\right| \approx 0.036 \mathrm{Cm}^{-2}$ for an aqueous solvent), $Z_{m}=10^{4}, n_{s}=10^{-4} \mathrm{M}$, a (fictive) equivalent volume fraction $\phi_{m} \equiv n_{m} \frac{4}{3} \pi a^{3}$ set to $10^{-3}$, (i.e., $n_{m} \approx 3.1714 \times 10^{-12} \mathrm{M}$ with $\mathrm{M}$ standing for mole per liter units). Thereby we have $\kappa_{0} a \simeq 16.4$. The profile of $E^{*}(z)$ reveals an unusual non-monotonic behavior near contact. This is a direct consequence of an accumulation of "excess" coions in the macroion depleted zone $(z<a)$ leading to a weaker screening.

In order to further characterize the adsorption behavior in the high screening regime, we examine $U_{m}$ as predicted by Eq. (77) and Eq. (9) for some typical experimental values of 


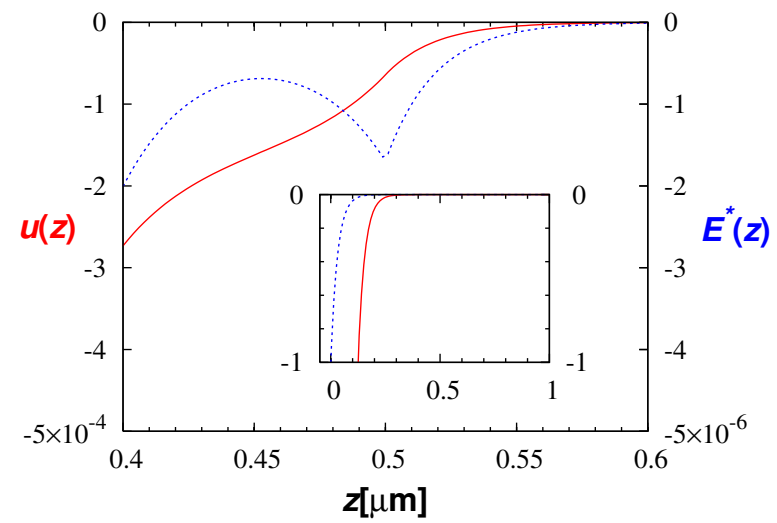

FIG. 2: Profiles of the reduced electrostatic potential $u(z)$ (solid lines) and the reduced electric field $E^{*}(z)=-\frac{b}{2} u^{\prime}(z)$ (dotted lines) around contact $(z=a=0.5 \mu \mathrm{m})$ calculated from Eqs. (2) and (31). The inset corresponds to a wider $z$-range. The following parameters were chosen: $b=10^{-3} \mu \mathrm{m}$, $\ell_{B}=7.14 \times 10^{-4} \mu \mathrm{m}, Z_{m}=10^{4}, n_{s}=10^{-4} \mathrm{M}$, and $\phi_{m}=10^{-3}$.

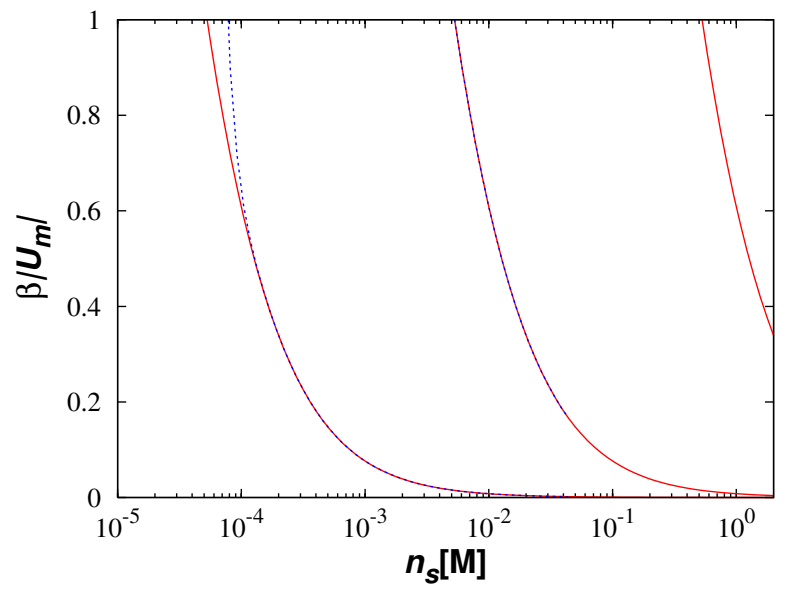

FIG. 3: Reduced contact potential of interaction $\beta\left|U_{m}\right|$ as a function of the molar bulk salt concentration $n_{s}$ in the strong screening regime with $a=0.5 \mu \mathrm{m}$ and $\phi_{m}=10^{-3}$. From left to right: $Z_{m}=10^{4}, 10^{5}$ and $10^{6}$. The solid lines were generated using Eq. (9). The dotted lines were generated using Eq. (7) with $b=1 \mathrm{~nm}$ and $\ell_{B}=0.714 \mathrm{~nm}$. For $Z_{m}=10^{6}$ the two curves are in practice identical.

the parameters $\kappa$ and $\kappa_{0}$ of charged colloidal systems. In Fig. 3, $-\beta U_{m}=\beta\left|U_{m}\right|$ is plotted against the bulk salt concentration $n_{s}$ for different prescribed values of $Z_{m}=10^{4}, 10^{5}$ and $10^{6}$. Only values of $\beta\left|U_{m}\right|$ smaller than unity are shown such as to explore its behavior 
where the DH approximation is valid. Figure 3 shows that beyond a certain threshold of salt content $n_{s}$ that is $Z_{m}$-dependent, no adsorption occurs as signaled by a nearly zero value of $U_{m}$. At relatively low enough salt concentration, adsorption is favored where $U_{m}$ increases with growing $Z_{m}$. The latter point can be clearly understood by noticing that for typical values of the parameters of charged colloidal systems $\left(Z_{m} \frac{n_{m}}{2 n_{s}} \ll 1\right.$ and $\left.Z_{m} \gg 1\right)$ we have $\beta U_{m} \approx 1-\sqrt{1+Z_{m}^{2} \frac{n_{m}}{2 n_{s}}}$. Moreover, in the limit $Z_{m}^{2} \frac{n_{m}}{2 n_{s}} \ll 1$, the simple following relation $\beta U_{m} \approx-Z_{m}^{2} \frac{n_{m}}{4 n_{s}}$ holds.

\section{B. Weak screening regime}

We now address the weak screening regime characterized by $\kappa_{0} a \ll 1$. In this situation Eq. (17) becomes:

$$
\beta U_{m} \simeq-\frac{2 Z_{m}}{\kappa b}\left[1+\frac{\kappa_{0}}{\kappa}\left\{\frac{\kappa b}{2 Z_{m}}\left(\frac{\kappa^{2}}{\kappa_{0}^{2}}-1\right)-1\right\} \kappa_{0} a\right],
$$

where the dispersion relation $\kappa^{2}=\kappa_{0}^{2}+Z_{m} \kappa_{c}^{2}$ has been used. Equation (10) reveals that $U_{m}$ varies affinely with macroion size $a$. Depending on the sign of $\frac{\kappa b}{2 Z_{m}}\left(\frac{\kappa^{2}}{\kappa_{0}^{2}}-1\right)-1, U_{m}$ may either decrease or increase with $a$. For $\frac{\kappa b}{2 Z_{m}} \gg 1$ we obtain the following limit behavior:

$$
\lim _{\frac{\kappa b}{2 Z_{m}} \rightarrow \infty} \beta U_{m} \approx-\left(1-\frac{\kappa_{0}^{2}}{\kappa^{2}}\right) \kappa a .
$$

In this "entropic" limit $\left|U_{m}\right|$ increases linearly with $a$, meaning that a finite wall-macroion attraction persists even at vanishing surface charge density $\sigma_{0}$ due to excluded volume effect $(a \neq 0)$. Noticing that

$$
E^{*}(a)=\frac{\kappa b}{2 Z_{m}} \beta U_{m}
$$

we deduce that the strength of the electric field at contact $\left|E^{*}(a)\right|$ becomes larger than unity when $\frac{\kappa b}{2 Z_{m}}$ is sufficiently large [especially true for Eq. (11)]. This feature corresponds to an electric field (or surface charge) amplification at contact.

Let us consider some typical experimental values of parameters representative of charged micellar systems (see caption of Fig. 4). Thereby we have $\kappa_{0} a \simeq 0.177$. To access the mechanisms of wall-macroion attraction in the weak screening regime, we have plotted the profiles of $u(z)$ and $E^{*}(z)$ in Fig. 4 for various values of $b$. Strikingly, in the macroion 


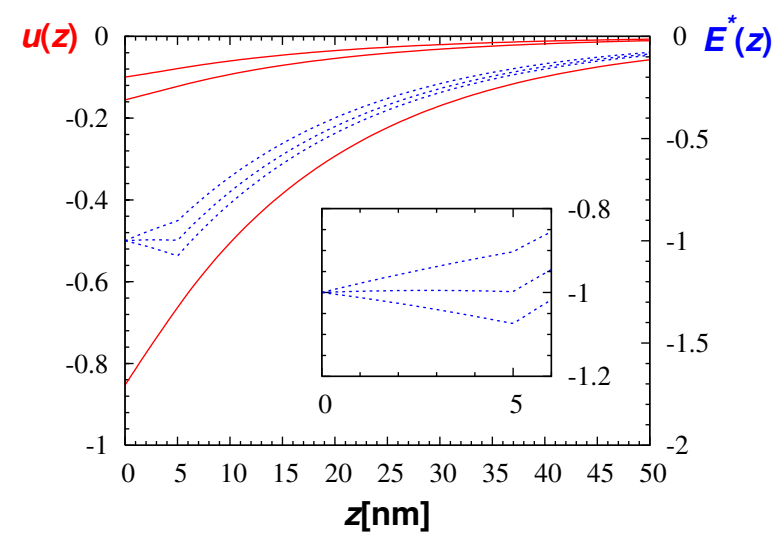

FIG. 4: Profiles of the reduced electrostatic potential $u(z)$ (solid lines - from top to bottom: $b=500,300,50 \mathrm{~nm}$ ) and the reduced electric field $E^{*}(z)=-\frac{b}{2} u^{\prime}(z)$ (dotted lines - from top to bottom: $b=50,300,500 \mathrm{~nm})$. The inset corresponds to a magnification of $E^{*}(z)$. The following parameters were chosen: $a=5 \mathrm{~nm}, \ell_{B}=0.714 \mathrm{~nm}, Z_{m}=10, n_{s}=10^{-4} \mathrm{M}$, and $\phi_{m}=10^{-3}$.

depleted zone, the electric field gets monotonically amplified (from $z=0$ up to contact $z=$ $a=5 \mathrm{~nm})$ at (very) large $b$ [corresponding to poorly charged interfaces $\left(\left|\sigma_{0}\right| \lesssim 10^{-4} \mathrm{Cm}^{-2}\right)$ - see Fig. 4. This phenomenon is again due to an accumulation of "excess" coions in the macroion depleted zone, that leads here to a net surface charge that is more negative than $\sigma_{0}$. It is important to remark, that surface charge amplification does not necessarily involve a subsequent charge reversal, as clearly indicated in Fig. 4. In fact this charge-amplification phenomenon was already observed, although uncommented, in computer simulations [see Fig. 4(b) in Ref. ${ }^{20}$ and Fig. 3 in Ref. ${ }^{21}$ ].

\section{CONCLUDING REMARKS}

To summarize, we have studied analytically within the framework of the Debye-Hückel theory the (weak) electrostatic adsorption of macroions at oppositely charged planar surfaces. Taking into account the crucial role of the finite size of the macroions, our model reveals non trivial adsorption driving forces. In the strong screening regime, the wallmacroion attraction strength at contact is exclusively governed by the screening strength contrast $\kappa / \kappa_{0}$. In the weak screening regime, the wall-macroion attraction strength can either decrease or increase with macroion size, depending on the surface charge density of the substrate. In particular, at sufficiently small surface charge densities an effective electric 
filed (or equivalently an effective surface charge) amplification sets in. All these adsorption mechanisms have a common feature, namely, the accumulation of excess coions in the macroion depleted zone. The latter mechanism also explains the surface charge amplification recently reported by molecular dynamics simulations for DNA-dendrimer complexation. ${ }^{21}$ Our findings could be experimentally verified by employing an (extra) ultra fine dispersion of charged particle tracers (with a smaller size than the macroions and such as to nearly not modify the screening strengths $\kappa$ and $\kappa_{0}$ ), whose density profile should reveal the electric filed' one in the macroion depleted zone.

\section{Acknowledgments}

The author thanks M. Lozada-Cassou for enlightening discussions. Financial support from DFG via LO418/12 and SFB TR6 is acknowledged.

* Electronic address: messina@thphy.uni-duesseldorf.de

1 H. Löwen and J.-P. Hansen, Annu. Rev. Phys. Chem. 51, 209 (2000).

2 Y. Levin, Rep. Prog. Phys. 65, 1577 (2002).

3 J. P. Hansen and I. McDonald, Theory of Simple Liquids (Academic, London, 1990).

4 G. Decher, Science 277, 1232 (1997).

5 H. Kawaguchi, Prog. Polym. Sci. 25, 1171 (2000).

6 W. Bu, D. Vaknin, and A. Travesset, Langmuir 22, 5673 (2006).

7 G. Luo et al., Science 13, 216 (2006).

8 G. L. Gouy, J. Phys. Radium 9, 457 (1910).

9 D. L. Chapman, Philos. Mag. 25, 475 (1913).

10 J. J. Spitzer, J. Colloid Interface Sci. 92, 198 (1983).

11 H. Greberg and R. Kjellander, J. Chem. Phys. 108, 2940 (1998).

12 R. Messina, E. González-Tovar, M. Lozada-Cassou, and C. Holm, Europhys. Lett. 60, 383 (2002).

13 J. Yu, G. E. Aguilar-Pineda, A. Antillón, S.-H. Dong, and M. Lozada-Cassou, J. Colloid Interface Sci. 295, 124 (2006). 
14 P. Gonzáles-Mozuelos and M. Medina-Noyola, J. Chem. Phys. 94, 1480 (1991).

15 R. R. Netz, Phys. Rev. E 60, 3174 (1999).

16 F. Jimenez-Ángeles and M. Lozada-Cassou, J. Phys. Chem. B 108, 7286 (2004).

17 J. J. Thomson, Philos. Mag 7, 237 (1904).

18 Note that the same result is obtained by requiring global elecroneutrality: $\int_{0}^{\infty} \sum_{\alpha} \rho_{\alpha}(z) d z=$ $-\sigma_{0}$.

19 Note that $\kappa^{2}=\kappa_{0}^{2}+Z_{m} \kappa_{c}^{2}$, so that $\kappa_{c}=\kappa_{c}\left(\kappa_{0}, \kappa, Z_{m}\right)$.

20 M. Tanaka and A. Y. Grosberg, J. Chem. Phys. 115, 567 (2001).

21 P. K. Maiti and B. Bagchi, Nano Letters 6, 2478 (2006). 